TRANSACTIONS OF THE

AMERICAN MATHEMATICAL SOCIETY

Volume 348 , Number 11, November 1996

\title{
ERRATUM À "DUALITÉ DANS LE GROUPE DE GROTHENDIECK DE LA CATÉGORIE DES REPRÉSENTATIONS LISSES DE LONGUEUR FINIE D'UN GROUPE RÉDUCTIF $p$-ADIQUE"
}

\author{
ANNE-MARIE AUBERT
}

Je remercie Guy Henniart et Marie-France Vignéras pour m'avoir signalé que la preuve de la proposition 3.2 de $[\mathrm{A}]$ est inexacte (je ne sais pas si cette proposition est vraie). La preuve dans $[\mathrm{A}]$ du théorème suivant ([A, th. 3.6]) utilise cette proposition et le but de cet erratum est de fournir une preuve différente de ce théorème. La démonstration présentée ici m’a été suggérée par Jean-Loup Waldspurger et je l'en remercie grandement.

Les notations non définies sont celles de $[\mathrm{A}]$. Soit $I \subset S$. Nous désignons par $\operatorname{Alg}(\{I\})$ la sous-catégorie abélienne de la catégorie des représentations lisses de $G$ constituée par les représentations de $G$ dont tout sous-quotient irréductible est sousquotient d'une représentation induite $\mathbf{i}_{L_{v(I)}}^{G}(\operatorname{Ad}(v)(\sigma))$, où $\sigma$ est une représentation irréductible cuspidale du sous-groupe de Levi standard $L_{I}$ de $G$ et $v \in W$ est tel que $v(I) \subset S \cap \Phi^{+}$(autrement dit, $L_{I}$ et $L_{v(I)}=v L_{I} v^{-1}$ sont des sous-groupes de Levi standard associés de $G$, et $\operatorname{Alg}(\{I\})$ ne dépend que la classe d'association de $\left.L_{I}\right)$.

Théorème. Pour tout $E$ dans $\operatorname{Alg}(\{I\})$, la suite

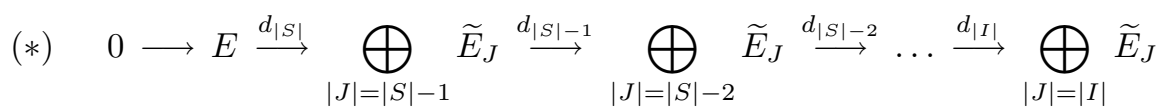

est exacte.

Démonstration. Par définition de la catégorie $\operatorname{Alg}(\{I\})$, l'exactitude de la suite $(*)$ est équivalente à celle, pour tout $I$ dans $\{I\}$, de la suite

$$
0 \longrightarrow E_{U_{I}} \stackrel{d_{|S|, I}}{\longrightarrow} \bigoplus_{|J|=|S|-1} \widetilde{E}_{J, I} \stackrel{d_{|S|-1, I}}{\longrightarrow} \bigoplus_{|J|=|S|-2} \widetilde{E}_{J, I} \stackrel{d_{|S|-2, I}}{\longrightarrow} \ldots \stackrel{d_{|I|, I}}{\longrightarrow} \bigoplus_{|J|=|I|} \widetilde{E}_{J, I},
$$

où $\widetilde{E}_{J, I}=\mathbf{r}_{L_{I}}^{G}\left(\widetilde{E}_{J}\right)$.

Soit $I \in\{I\}$ fixé. Pour $J \subset S$, le terme $\widetilde{E}_{J, I}$ est filtré de la manière suivante. Notons $\Theta$ l'ensemble des parties $\theta$ de $W$ telles que, si $w \in \theta$ et $l(w)<l\left(w^{\prime}\right)$, alors $w^{\prime} \in \theta$. Soit $\Theta_{J} \subset \Theta$ l'ensemble des $\theta \in \Theta$ qui sont stables par multiplication à gauche par $W_{J}$. Pour tout $\theta \in \Theta_{J}$, nous posons $G_{\theta}:=\bigcup_{w \in \theta} B w P_{I}$ et nous notons $\widetilde{E}_{J}^{\theta}$ le sous-module de $\widetilde{E}_{J}$ formé des éléments de $\widetilde{E}_{J}$ à support compact contenu

Received by the editors April 11, 1996.

1991 Mathematics Subject Classification. Primary 20G40, 22E50.

(C)1996 American Mathematical Society 
dans $G_{\theta}$. Pour $\theta$ élément quelconque de $\Theta$, nous posons $\widetilde{E}_{J}^{\theta}:=\widetilde{E}_{J}^{\theta^{\prime}}$, où $\theta^{\prime}$ est le plus grand élément de $\Theta_{J}$ contenu dans $\theta$.

Si $\theta_{1} \in \Theta$ et $\theta_{2} \in \Theta$ sont telles que $\theta_{1} \subset \theta_{2}$, on a $\widetilde{E}_{J}^{\theta_{1}} \subset \widetilde{E}_{J}^{\theta_{2}}$. Pour $f \in \widetilde{E}_{J}^{\theta_{2}}$, nous notons $\operatorname{Res}_{\theta_{2}-\theta_{1}}^{\theta_{2}}(f)$ la restriction de $f$ à $G_{\theta_{2}-\theta_{1}}$. La suite

$$
0 \longrightarrow \widetilde{E}_{J}^{\theta_{1}} \longrightarrow \widetilde{E}_{J}^{\theta_{2}} \stackrel{\operatorname{Res}_{\theta_{2}-\theta_{1}}^{\theta_{2}}}{\longrightarrow} \widetilde{E}_{J}^{\theta_{2}-\theta_{1}} \longrightarrow 0
$$

est exacte (voir par exemple [C, lem. 6.1.1.]); l'application $\operatorname{Res}_{\theta_{2}-\theta_{1}}^{\theta_{2}}$ de $\widetilde{E}_{J}^{\theta_{2}} / \widetilde{E}_{J}^{\theta_{1}}$ dans $\widetilde{E}_{J}^{\theta_{2}-\theta_{1}}$, définie par $\operatorname{Res}_{\theta_{2}-\theta_{1}}^{\theta_{2}}\left(f+\widetilde{E}_{J}^{\theta_{1}}\right):=\operatorname{Res}_{\theta_{2}-\theta_{1}}^{\theta_{2}}(f)$ pour $f \in \widetilde{E}_{J}^{\theta_{2}}$, est donc un isomorphisme.

Si $\theta_{1} \subset \theta_{2}$ et $\theta_{1}^{\prime} \subset \theta_{2}^{\prime}$ (éléments de $\Theta_{J}$ ) vérifient $\theta_{1} \subset \theta_{1}^{\prime}$ et $\theta_{2} \subset \theta_{2}^{\prime}$ avec $\theta_{2}-\theta_{1}=\theta_{2}^{\prime}-\theta_{1}^{\prime}$, alors le diagramme suivant est commutatif

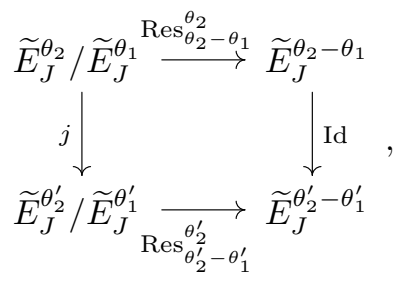

où $j\left(f+\widetilde{E}_{J}^{\theta_{1}}\right):=f+\widetilde{E}_{J}^{\theta_{1}^{\prime}}$, pour $f \in \widetilde{E}_{J}^{\theta_{2}}$

S'il existe $w \in W$ de longueur minimale dans la double classe $W_{J} w W_{I}$ tel que $\theta_{2}-\theta_{1}=W_{J} w$, on a $B W_{J} w P_{I}=P_{J} w P_{I}$ et l'application de $\left(\mathbf{i}_{L_{J}}^{P_{J} w P_{I}} \circ \mathbf{r}_{L_{J}}^{G}\right)(E)$ dans $\left(\mathbf{i}_{w^{-1} P_{J} w \cap P_{I}}^{P_{I}} \circ \operatorname{Ad}\left(w^{-1}\right) \circ \mathbf{r}_{L_{J}}^{G}\right)(E)$ définie par $f \mapsto \Phi_{f}$ avec $\Phi_{f}(p)=f(w p)$ pour $p \in P_{I}$ est un isomorphisme (i désigne ici le foncteur induction à support compact normalisé).

Le groupe $w^{-1} P_{J} w \cap L_{I}$ est le sous-groupe parabolique standard de $L_{I}$ associé à $w^{-1}(J) \cap I$, il a comme radical unipotent $w^{-1} U_{J} w \cap L_{I}$ et comme sous-groupe de Levi $w^{-1} L_{J} w \cap L_{I}$. Notons alors $\xi \mapsto \bar{\xi}$ l'application canonique de $E_{U_{J}}=\mathbf{r}_{L_{J}}^{G}(E)$ $\operatorname{sur}\left(E_{U_{J}}\right)_{U_{J} \cap w U_{I} w^{-1}}=\mathbf{r}_{w L_{I} w^{-1} \cap L_{J}}^{L_{J}}\left(E_{U_{J}}\right)$. D'après [C, prop. 6.2.1 et 6.3.3] l'application de $\left(\left(\mathbf{i}_{w^{-1} P_{J} w \cap P_{I}}^{P_{I}} \circ \operatorname{Ad}\left(w^{-1}\right) \circ \mathbf{r}_{L_{J}}^{G}\right)(E)\right)_{U_{I}}$ dans

$$
\left(\mathbf{i}_{L_{I} \cap w^{-1} L_{J} w}^{L_{I}} \circ \operatorname{Ad}\left(w^{-1}\right) \circ \mathbf{r}_{w L_{I} w^{-1} \cap L_{J}}^{L_{J}}\right)\left(\mathbf{r}_{L_{J}}^{G}(E)\right)
$$

qui à $\Phi_{f}$ associe

$$
l \mapsto \int_{U_{I} \cap w^{-1} P_{J} w \backslash U_{I}} \overline{\Phi_{f}(u l)} \mathrm{d} \mu(u)
$$

est un isomorphisme. Mais, le terme $\left(\mathbf{i}_{L_{I} \cap w^{-1} L_{J} w}^{L_{I}} \circ \operatorname{Ad}\left(w^{-1}\right) \circ \mathbf{r}_{w L_{I} w^{-1} \cap L_{J}}^{L_{J}}\right)\left(\mathbf{r}_{L_{J}}^{G}(E)\right)$ est égal à $\left(\mathbf{i}_{L_{I} \cap w^{-1} L_{J} w^{L_{I}}} \circ \mathrm{Ad}\left(w^{-1}\right) \circ \mathbf{r}_{w L_{I} w^{-1} \cap L_{J}}^{G}\right)(E)$, par transivité du foncteur $\mathbf{r}_{L_{J}}^{G}$ (voir par exemple [BZ, prop. 2.3 (c)]); comme $E$ est dans la catégorie $\operatorname{Alg}(\{I\})$, si $\mathbf{r}_{w L_{I} w^{-1} \cap L_{J}}^{G}(E)$ est non nul, il existe $v \in W$ tel que $w L_{I} w^{-1} \cap L_{J}=v L_{I} v^{-1}$, le groupe $v L_{I} v^{-1}$ est alors contenu dans (et par conséquent lui est égal) $w L_{I} w^{-1}$, il s'ensuit que $w L_{I} w^{-1}$ est alors contenu $L_{J}$ et l'on a alors

$$
\left(\mathbf{i}_{L_{I} \cap w^{-1} L_{J} w}^{L_{I}} \circ \operatorname{Ad}\left(w^{-1}\right) \circ \mathbf{r}_{w L_{I} w^{-1} \cap L_{J}}^{G}\right)(E)=\left(\operatorname{Ad}\left(w^{-1}\right) \circ \mathbf{r}_{w L_{I} w^{-1}}^{G}\right)(E) .
$$

Pour $\theta$ élément de $\Theta$, nous notons $\widetilde{E}_{J, I}$ l'image dans $\widetilde{E}_{J, I}$ de $\widetilde{E}_{J}^{\theta}$. Si $\theta_{1} \subset \theta_{2}$, par exactitude $\mathrm{du}$ foncteur de Jacquet $\mathbf{r}_{L_{I}}^{G}$, on a $\widetilde{E}_{J, I}^{\theta_{2}} / \widetilde{E}_{J, I}^{\theta_{1}}=\widetilde{E}_{J, I}^{\theta_{2}-\theta_{1}}$. Ce qui 
précède montre que, s'il existe $w \in W$ de longueur minimale dans la double classe $W_{J} w W_{I}$ tel que $\theta_{2}-\theta_{1}=W_{J} w$, et si $\widetilde{E}_{J, I}^{\theta_{2}} / \widetilde{E}_{J, I}^{\theta_{1}}$ est non nul, on a $w L_{I} w^{-1} \subset L_{J}$ et l'application

$$
f \mapsto\left(l \mapsto \int_{U_{I} \cap w^{-1} P_{J} w \backslash U_{I}} \overline{f(w u l)} d \mu(u)\right)
$$

définit un isomorphisme de $\left(\left(\mathbf{i}_{L_{J}}^{G_{W_{J}} w} \circ \mathbf{r}_{L_{J}}^{G}\right)(E)\right)_{U_{I}} \operatorname{sur}\left(\operatorname{Ad}\left(w^{-1}\right) \circ \mathbf{r}_{w L_{I} w^{-1}}^{G}\right)(E)$.

Rappelons que, pour $K \subset J$, on note $\pi_{K}^{J}$ la surjection canonique de $E_{U_{J}}$ sur $E_{U_{K}}=E_{U_{J}} / E_{U_{J}}\left(U_{K} \cap L_{J}\right)$ et $\varphi_{K}^{J}$ l'application de $E_{J}$ dans $E_{K}$ qui à $f$ associe $g \mapsto \pi_{K}^{J}(f(g))$. Les applications de transition $d_{k, U_{I}}$ étant des sommes avec signes d'applications $\varphi_{K}^{J}$, elles respectent la filtration par $\Theta$, i.e., pour tout $\theta \in \Theta$ et tout $k \in\{1, \ldots,|S|\}$, on a

$$
d_{k, U_{I}}\left(\bigoplus_{|J|=k} \widetilde{E}_{J, I}^{\theta}\right) \subset \bigoplus_{|J|=k-1} \widetilde{E}_{J, I}^{\theta}
$$

Choisissons une suite $\theta_{1} \supset \theta_{2} \supset \cdots \supset \theta_{t+1}$ d'éléments de $\Theta$, telle que $\theta_{1}=W$, $\theta_{t+1}=\emptyset$ et $\theta_{i}-\theta_{i+1}=w_{i}$ avec $w_{i} \in W$. Il suffit donc de prouver que, pour tout $i \in\{1, \ldots, t\}$, la suite des gradués

$$
0 \longrightarrow E_{U_{I}}^{\theta_{i}} / E_{U_{I}}^{\theta_{i+1}} \stackrel{d_{|S|, I}^{\theta_{i}}}{\longrightarrow} \bigoplus_{|J|=|S|-1} \widetilde{E}_{J, I}^{\theta_{i}} / \widetilde{E}_{J, I}^{\theta_{i+1}} \stackrel{d_{|S|-1, I}^{\theta_{i}}}{\longrightarrow} \ldots \stackrel{d_{|I|, I}^{\theta_{i}} \longrightarrow}{\longrightarrow} \bigoplus_{|J|=|I|} \widetilde{E}_{J, I}^{\theta_{i}} / \widetilde{E}_{J, I}^{\theta_{i+1}}
$$

est exacte. Fixons $i$ et posons $w:=w_{i}$. Notons $\theta_{i}^{\prime}$ (resp. $\theta_{i+1}^{\prime}$ ) le plus grand élément de $\Theta_{J}$ qui est contenu dans $\theta_{i}$ (resp. $\theta_{i+1}$ ). Supposons $\widetilde{E}_{J, I}^{\theta_{i}} / \widetilde{E}_{J, I}^{\theta_{i+1}}$ est non nul. Alors $\theta_{i}^{\prime}$ et $\theta_{i+1}^{\prime}$ sont distincts et la classe à gauche $W_{J} w$ est donc contenue dans $\theta_{i}$. Par définition des ensembles $\theta$, les éléments de $\theta_{i+1}$ (et donc de $\theta_{i}$ ) sont de longueur inférieure à la longueur de $w$; par conséquent l'inclusion $W_{J} w \subset \theta_{i}$ montre que $w$ est de longueur minimale dans $W_{J} w$, autrement dit, que $w^{-1} \alpha>0$ pour tout $\alpha \in J$. D'autre part, il résulte de ce qui précède que $w W_{I} w^{-1}$ est contenu dans $W_{J}$ (car $E$ appartient à $\left.\operatorname{Alg}(\{I\})\right)$ et que $\widetilde{E}_{J, I}^{\theta_{i}} / \widetilde{E}_{J, I}^{\theta_{i+1}}$ est isomorphe à $\left(\operatorname{Ad}\left(w^{-1}\right) \circ \mathbf{r}_{w L_{I} w^{-1}}^{G}\right)(E)$. Puisque $w$ est de longueur minimale dans $W_{J} w$, il est donc aussi de longueur minimale dans $w W_{I}$. Il s'ensuit que l'ensemble $w(I)$ est formé de racines simples et est contenu dans $J$. On est ramené à montrer que la suite

$$
\cdots \longrightarrow \bigoplus_{\substack{|J|=k \\ w(I) \subset J \subset S^{w}}} \operatorname{Ad}\left(w^{-1}\right)\left(\mathbf{r}_{w L_{I} w^{-1}}^{G} E\right) \stackrel{\delta_{k}}{\longrightarrow} \bigoplus_{\substack{|J|=k-1 \\ w(I) \subset J \subset S^{w}}} \operatorname{Ad}\left(w^{-1}\right)\left(\mathbf{r}_{w L_{I} w^{-1}}^{G} E\right) \stackrel{\delta_{k-1}}{\longrightarrow} \ldots
$$

(où $S^{w}:=S_{w^{-1}}=\left\{\alpha \in S \mid w^{-1} \alpha>0\right\}$, notation de [A, p. 2182]) est exacte. Il résulte de la transivité du foncteur $\mathbf{r}_{L}^{G}$ (voir par exemple [BZ, prop. 2.3 (c)]) que, pour $I, J$ et $K$ des parties de $S$ telles que $w(I) \subset J$ et $w(I) \subset K \subset J$, le diagramme suivant est commutatif:

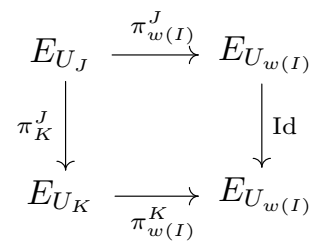


La construction de l'isomorphisme de $\widetilde{E}_{J, I}^{\theta_{i}} / \widetilde{E}_{J, I}^{\theta_{i+1}} \operatorname{sur} \operatorname{Ad}\left(w^{-1}\right)\left(\mathbf{r}_{w L_{I} w^{-1}}^{G} E\right)$ montre alors que les applications de transitions $\delta_{k}$ sont l'identité de $\operatorname{Ad}\left(w^{-1}\right)\left(\mathbf{r}_{w L_{I} w^{-1}}^{G} E\right)$ multipliée par le signe qu'il faut. La suite ci-dessus est donc le produit tensoriel de $\operatorname{Ad}\left(w^{-1}\right)\left(\mathbf{r}_{w L_{I} w^{-1}}^{G} E\right)$ et du complexe de Koszul $\Lambda^{\cdot}\left(\mathbb{C}^{S^{w}-w(I)}\right)$ translaté de $|S|-\left|S^{w}\right|$. Il est bien connu que ce complexe est acyclique sauf si $S^{w}=w(I)$ (voir par exemple [S, chap. IV.A]). Mais si $S^{w}=w(I)$, la suite se réduit à

$$
0 \longrightarrow 0 \longrightarrow \ldots \longrightarrow 0 \longrightarrow \operatorname{Ad}\left(w^{-1}\right)\left(\mathbf{r}_{w L_{I} w^{-1}}^{G} E\right)
$$

qui est encore exacte.

Remarques. (1) Si $w \in W$ est tel que $w(I) \subset \Phi^{+}$et $S^{w}=w(I)$, alors $w(S-I) \subset$ $-\Phi^{+}$(voir [A, bas de la page 2182]) et, par conséquent, $w^{-1}$ est égal à l'élément de plus grande longueur dans $\mathcal{D}(I, \emptyset)$, élément noté $w_{I}$ dans $[\mathrm{A}]$.

Nous désignons par $\widetilde{D}_{G}$ l'application qui à $E \in \operatorname{Alg}(\{I\})$ associe le conoyau $E^{\#}$ de l'application $d_{|I|}$. Pour tout $E \in \operatorname{Alg}(\{I\})$, on a $\widetilde{D}_{L_{I}}\left(\mathbf{r}_{L_{I}}^{G}(E)\right)=\mathbf{r}_{L_{I}}^{G}(E)$. Le calcul précédent, appliqué au dernier cran, calcule $\left(\widetilde{D}_{G}(E)\right)_{U_{I}}=\left(\mathbf{r}_{L_{I}}^{G} \circ \widetilde{D}_{G}\right)(E)$ :

$(* *) \quad\left(\mathbf{r}_{L_{I}}^{G} \circ \widetilde{D}_{G}\right)(E)=\left(\operatorname{Ad}\left(w_{I}\right) \circ \widetilde{D}_{L_{I^{\prime}}} \circ \mathbf{r}_{L_{I^{\prime}}}^{G}\right)(E)$, pour tout $E \in \operatorname{Alg}(\{I\})$, où $I^{\prime}=w_{I}^{-1}(I)$.

L'égalité $(* *)$ et le fait que $\left[\widetilde{D}_{G}(E)\right]=(-1)^{|I|} D_{G}([E])$ (voir $[$ A, cor. 3.9 (a)]) montrent que $\left(\mathbf{r}_{L_{I}}^{G} \circ D_{G}\right)(E)=\left(\operatorname{Ad}\left(w_{I}\right) \circ D_{L_{I^{\prime}}} \circ \mathbf{r}_{L_{I^{\prime}}}^{G}\right)(E)$, égalité qui est un cas particulier de [A, th. $1.7(2)]$.

(2) J. Bernstein a aussi annoncé le théorème prouvé ici (colloque Luminy 1994). Dans le cas des représentations lisses de longueur finie une preuve de nature différente de notre théorème figure dans [P. Schneider et U. Stuhler, Representation theory and sheaves on the Bruhat-Tits building, Prépublication 1995, section IV $\S 5]$.

\section{REFERENCES}

[A] A.-M. Aubert, Dualité dans le groupe de Grothendieck de la catégorie des représentations lisses de longueur finie d'un groupe réductif p-adique, Trans. Amer. Math. Soc. 347 (1995), 2179-2189. MR 91i:22025

[B] J.N. Bernstein (rédigé par P. Deligne), Le "centre" de Bernstein, Représentations des groupes réductifs sur un corps local, Travaux en cours, Hermann, Paris, 1984. MR 86e:22028

[BZ] J.N. Bernstein et A. Zelevinski, Induced representations of reductive p-adic groups I, Ann. Sci. Ecole. Norm. Sup. 10 (1977), 441-472. MR 58:28310

[C] W. Casselman, Introduction to the theory of admissible representations of $p$-adic reductive groups, Prépublication 1974 (Nouvelle version Mai 1995).

[S] J.-P. Serre (Cours au Collège de France 1957-1958, rédigé par P. Gabriel), Algèbre Locale. Multiplicités, Lecture Notes in Math., vol. 11, Springer (deuxième édition), BerlinHeidelberg-New-York, 1965. MR 34:1352

Ecole Normale Supérieure, L.M.E.N.S.-D.M.I. (C.N.R.S. U.R.A. 762), 45 Rue D'Ulm, F-75005 PARIS, France

E-mail address: aubert@dmi.ens.fr 